\title{
Marked Reduction in 28-Day Mortality among Elderly Patients with Severe Community-Acquired Pneumonia: Post Hoc Analysis of XueBiJing Injection Randomized Controlled Trial
}

Yan Liu

Beijing University of Chinese Medicine Affiliated Dongzhimen Hospital

Chi Zhang ( $\sim 13552695467 @ 126 . c o m$ )

Beijing University of Chinese Medicine Affiliated Dongzhimen Hospital

Chengyu Li

Beijing University of Chinese Medicine

Chunxue Bai

Fudan University

Hongcai Shang

Beijing University of Chinese Medicine Affiliated Dongzhimen Hospital

\section{Research}

Keywords: Severe community-acquired pneumonia, randomized controlled trial, post hoc analysis, 28-day mortality rate, XueBiJing injection

Posted Date: March 5th, 2020

DOI: https://doi.org/10.21203/rs.3.rs-16216/v1

License: (c) (i) This work is licensed under a Creative Commons Attribution 4.0 International License. Read Full License 


\section{Abstract}

Background There were a few studies on the case mortality of severe community-acquired pneumonia (CAP) in elderly people. Improved outcomes with XueBiJing (XBJ) Injection versus placebo have been shown in overall trial populations. We investigated the efficacy and safety of XBJ versus placebo in subjects with severe CAP stratified by age ( $<65$ and $\geq 65$ years).

Methods This post hoc analysis of a large randomized trial compared data from elder and nonelderly patients with XBJ, $100 \mathrm{ml}$, q12h, or a visually indistinguishable placebo for 5-7 days.

Results Among subjects $\geq 65$ years ( $n=291), 23$ (16.0\%) XBJ recipients and 41 (27.9\%) placebo recipients $(P=0.014)$ died within 28 days. Among subjects $<65$ years $(n=360)$, XBJ still had lower mortality (XBJ 15.6\% verse placebo $22.8 \% ; P=0.082$ ), without significantly statistical difference. Total duration of ICU stay and the time of mechanical ventilation were similar in both groups $(P>0.05)$. XBJ also had a favorable safety profile, with no clinically relevant differences between two groups. The overall incidence of adverse events was similar in both groups.

Conclusions XBJ was safe and effective for reduction in 28-day mortality among elderly patients with severe CAP. Additional trials involving elderly patients are needed to further confirm the present results.

\section{Background}

Community-acquired pneumonia (CAP) represents an important threat to the health of elder adults[1]. Like other respiratory infections, people at the extremes of age are at greatest risk and have worse outcomes. In adults, the incidence of CAP and related hospitalization and mortality increase steadily with age, even when these rates are adjusted for chronic health problems such as lung or heart conditions[2, 3]. Specially, the incidence of CAP in adults increases with age, with a dramatic rise after age 65. In developed countries, almost one half of the total hospitalizations for CAP occur in patients over 65 years and pneumonia is a leading cause of death among this age group[4]. The severity of CAP also increases with age, primarily due to age-related immune dysfunction, and greater likelihood of underlying comorbid factors in elderly patients[5].

However, despite the recognized importance of CAP in the elderly, there is little information about the precise etiology and prognosis factors affecting elder patients admitted to an intensive care unit (ICU) for severe CAP although such knowledge seems basic to the most appropriate management of elder patients[6]. American Thoracic Society guidelines suggest that three criteria can be used to define appropriate empirical treatment of CAP: the severity of pneumonia at presentation, the presence of underlying disease, and age [7]. As a result, different antimicrobial strategies are proposed for outpatients according to age and/or underlying condition. Conversely, all patients with severe CAP are included in the same therapeutic group whatever their age, and the potential implications of advanced age on antimicrobial management are not mentioned. Given the morbidity and mortality of severe CAP in the elderly, new therapeutic options would be valuable[8].

XueBiJing (XBJ), an herbal-based intravenous preparation approved by the National Medical Products Administration (NMPA) China in 2004, has been incorporated into routine sepsis care in China $[9,10]$. Pharmacological studies have shown that XBJ has an antagonistic effect on endotoxin, and an inhibitory effect on the uncontrolled release of endogenous inflammatory mediators produced by endotoxin-stimulated monocytes/macrophages [11-13]. A recent well-done randomized study showed XBJ effective in patients with severe CAP [14]. Impressive benefit with this herbal-based medication was an $8.8 \%$ absolute reduction in mortality in patients who received XBJ. 
Few clinical trials have examined the safety or impact of new therapeutic options on mortality rate in an elder highrisk cohort with severe CAP, data in individuals with severe CAP in this age group is largely lacking. The present analysis evaluated the effects of XBJ and placebo in two age groups ( $\geq 65,<65$ years) using data from the XBJ trial. Subjects aged 65 years may represent elder patients of greatest concern to clinicians. This study provides information to clinicians on the efficacy and safety of XBJ in the elderly subjects with severe CAP.

\section{Methods}

\section{Design Overview}

A post-hoc analysis was carried out using data from the main multicenter randomized controlled

trial (RCT) "XueBiJing injection versus placebo for critically ill patients with severe community-acquired pneumonia: a randomized controlled trial". Details of the XBJ trial have been previously published. The protocol is consistent with the principles of the Declaration of Helsinki, was approved by the Medical Ethics Committee of Zhongshan Hospital, Fudan University [2011-38(3)] and the participants gave their written informed consent. The trial included 710 severe CAP patients, randomly assigned to receive XBJ $(n=334)$, or placebo $(n=341)$. The participants received the solvent only (normal saline, $200 \mathrm{ml}, \mathrm{q} 12 \mathrm{~h}$ ) in the placebo group and the solvent plus XBJ (normal saline $100 \mathrm{ml}+\mathrm{XBJ} 100 \mathrm{ml}$, q12h) in the XBJ group. Both groups received a standard therapy (such as antibiotics) chosen by the attending physician according to the 2007 ATS/IDSA guideline. The primary outcome was 8-day improvement in the pneumonia severity index (PSI) risk rating. Main secondary outcomes were 28-day mortality rate, duration of mechanical ventilation and total duration of ICU stay. In this post hoc analysis of the XBJ study the primary outcome was 28-day mortality. Other secondary endpoints were the time of mechanical ventilation, total duration of ICU stay.

\section{Statistical Analyses}

Primary outcome analysis was a simple categorical frequency comparison by use of the $\chi^{2}$ test. For time-to-event variables, Kaplan-Meier estimates were used and the groups were compared with a log-rank test. Hazard ratio (HR) and associated 95\% confidence intervals (Cls) were estimated from the Cox proportional hazards model.

The secondary outcome for the time of mechanical ventilation was analyzed by t test or the Wilcoxon rank sum test as appropriate. The same analysis was used for other continuous variables, such as total duration of ICU stay. Categorical variables were compared using the chi-square test or Fisher exact test. Descriptive statistics (number and frequency) were used to summarize all safety outcomes for each group. Safety outcomes included serious, nonserious adverse events, and laboratory measurements.

All outcomes were analyzed in the intent-to treat population, which included all patients as randomized. Statistical analyses were performed using SAS version 9.4 (SAS Institute Inc.), with a 2-sided P value of less than .05 considered significant.

\section{Results}

Demographic characteristics stratified according to age group and treatment arms are summarized in Table 1. The mean age of elder patients ( $n=291$ ) was $70.47 \pm 3.23$ years; and $n=190,65.29 \%$ were male. The mean age of nonelderly patients $(n=360)$ was $49.76 \pm 11.63$ years; and $n=253,70.28 \%$ were male. The mean BMl at baseline did not differ between treatments or age groups ( $\geq 65$ years $P=0.929,<65$ years $P=0.749$ ). Differences between age groups in baseline comorbidities, PSI score or other biological parameters were not significant. 
Rate of patients with acute respiratory distress syndrome (ARDS) and septic shock, the baseline settings of mechanical ventilation, and the frequency of antimicrobial prescriptions in each age group are listed in Table 2. There were no significant differences in antimicrobial treatment (Beta-lactam, quinolones, glycopeptide, oxazolidinones, antifungal agents, tetracyclines, macrolide, aminoglycoside, nitroimidazoles, antivirals, lincomycin, and sulfanilamide) or patient parameters (septic shock and ARDS at baseline and on study) between the XBJ and placebo groups in each age group. In addition, microbiologic identification was similar in both study groups (all $P>0.05$ )

(TABLE S1).

Among elder patients ( $n=291), 28$-day mortality was significantly lower in those who received XBJ verse those who received placebo $(P=0.014)$. No statistically significant difference in mortality between two groups was seen in younger patients $(P=0.082)$. Both elderly and nonelderly patients experienced shorter the time of mechanical ventilation and total duration of ICU stay, however, without significantly differences. Some secondary outcomes are listed in the Table S2. Among elder patients, the XBJ group had a significantly lower pneumonia severity index (PSI) $(96.0 \pm 26.18)$ than the control group $(106.9 \pm 28.92)(P=0.001)$ at day 8 .

Adverse events (AEs) that occurred with a frequency of $>1 \%$ are summarized in Table S3. Overall, XBJ demonstrated a similar overall safety profile among the subgroups of patients aged $<65$ and $\geq 65$ years. In particular, very similar incidences of low red blood cell count (7.22\% vs. 5.56\%) and elevated aspartate amino transferase (5.56\% vs. $7.64 \%)$ were seen in the subgroups aged $<65$ and $\geq 65$ years, respectively. There were no severe AEs that occurred in either elder or nonelderly patients after XBJ therapy.

\section{Discussion}

The overall results of this large trial showed that XBJ is an effective and well-tolerated therapy for patients with severe CAP [14]. With high efficacy and good tolerability, XBJ compares favorably with the placebo. Especially, there was an $8.8 \%$ absolute reduction in mortality in patients who received XBJ. In addition, with improved PSI risk score.

In the current analysis, XBJ showed a similar favorable safety profile in elder and younger patients alike, and the high efficacy of XBJ was preserved in elder patients. No clinically relevant differences in safety profile were observed between the subgroups of patients aged $<65$ years and $\geq 65$ years. These results suggest that there is no a priori need for upfront dose reductions when prescribing XBJ to elderly patients. Prospectively planned ongoing trial is further evaluating the efficacy and safety benefits of XBJ in elder patients.

CAP seems more severe in elder than in younger patients. Fine and colleagues' have demonstrated that age over 65 years was one of the five predisposing factors for a complicated course. In most prognosis studies, age is a significant predictor of mortality [15]. Consequently, the mortality rate of such pulmonary infection seems high in elder people. Among elder hospitalized patients overall, the death rate was about $30 \%[16,17]$. When patients were admitted to an ICU, mortality was even higher [18]. In Rello's study, the mortality rate was 40\% [18]. In the present work, $94.07 \%$ of the participants were from ICUs, the 28 day mortality rate among elder patients with XBJ was $16.0 \%$. This rate was significantly lower than the rate observed in placebo group (27.9\%).

Although efficacy was not the primary end point of the original study, efficacy comparisons were undertaken to allow for a more clinically meaningful comparison of the tolerability profiles and to explore any clinically relevant differences in efficacy between younger and elder patients. One recently presented study has evaluated hydrocortisone in patients with severe CAP in the ICU setting [19]. Among patients with mean age 62 years, a 
continuous infusion of hydrocortisone did not result in lower 28-day mortality than placebo. We believe that this analysis is particularly intriguing: in fact, this result on mortality overcomes one of the main limitations of our previous study.

All herbal therapy requires active supervision, particularly for elderly patients, who could have other illnesses and be receiving concomitant medications[20]. Elder patients could also be at greater risk of experiencing toxicity, particularly if their renal function is impaired. Unlike tablets at home, XBJ I.V. therapy is administered in the hospitals and the clinicians has an active role in treatment administration and the management of any toxicity.

The interpretation of this post hoc analysis has limitations to be considered. First, neither study was specifically designed to assess efficacy or in safety exclusively elder patients. Furthermore, the number of patients included in each age group was relatively small and did not allow multivariate analysis involving backward stepwise logistic regression in each group to show the exact level of significance.

\section{Conclusions}

In conclusion, XBJ treatment demonstrated similar effects in the subgroup of elder patients with severe CAP. This post-hoc analysis represents a proof-of concept study, and, this analysis found a differential effect of age on XBJ treatment in patients with severe CAP. Thus, this proof-of concept study needs to be confirmed by a large, well designed, and appropriately focused randomized clinical trial in an elder population.

\section{Abbreviations}

AE

adverse event

APACHE

acute physiology and chronic health evaluation

ARDS

acute respiratory distress syndrome

CAP

community-acquired pneumonia

Cls

confidence intervals

ICU

intensive care unit

K-M curve

kaplan-meier curve

NMPA

national medical products administration

PSI

pneumonia severity index

RCT

randomized controlled trial

SIRS

systemic inflammatory response syndrome

SOFA 
sequential organ failure assessment

XBJ

Xuebijing injection

\section{Declarations}

Ethics approval and consent to participate The protocol is consistent with the principles of the Declaration of Helsinki, was approved by the Medical Ethics Committee of Zhongshan Hospital, Fudan University [2011-38(3)] and the participants gave their written informed consent.

Consent for publication Not applicable.

Availability of data and materials All data generated or analyzed during this study are included in this published article [XueBiJing Injection Versus Placebo for Critically III Patients With Severe Community-Acquired Pneumonia: A Randomized Controlled Trial. Critical Care Medicine, 2019, 47(9):1.].

Competing interests: The authors declare that they have no competing interests.

Funding This work was supported by National High-level Personnel of Special Support Program (W02020052).

Authors' contributions Yan Liu and Chi Zhang contributed equally to this work, they finished acquisition and analysis of the data, as well as wrote the manuscript. Chengyu Li participated and performed data analysis in this work.

Hongcai Shang and Chunxue Bai provided critical revisions of the manuscript. All members of the writing committee read and approved the final report.

Acknowledgements The authors appreciate all the participants for making contributions to this work.

\section{Author information}

Equal Contributors; Yan Liu and Chi Zhang are equally contributing co-first authors.

\section{Affiliations}

Key Laboratory of Chinese Internal Medicine of Ministry of Education and Beijing, Dongzhimen Hospital, Beijing University of Chinese Medicine, Beijing, China.

Yan Liu, Chengyu Li, Hongcai Shang

Institute of Neurological Disorders and Stroke, Dongzhimen Hospital, Beijing University of Chinese Medicine, Beijing, China.

Chi Zhang

Department of Respiratory Medicine, Zhongshan Hospital, Fudan University, Shanghai, China.

Chunxue Bai

\section{Tables}


TABLE 1. Comparison of Demographic and Basal Clinical Characteristics of Patients Between XueBiJing Injection and Placebo Groups 


\begin{tabular}{|c|c|c|c|c|c|c|}
\hline \multirow[t]{2}{*}{ Characteristics } & \multicolumn{3}{|c|}{ Elder $\geq 65$} & \multicolumn{3}{|c|}{ Nonelderly 065} \\
\hline & $\begin{array}{l}\text { Placebo } \\
\text { Group }\end{array}$ & $\begin{array}{l}\text { Xuebijing } \\
\text { Group }\end{array}$ & value & $\begin{array}{l}\text { Placebo } \\
\text { Group }\end{array}$ & $\begin{array}{l}\text { Xuebijing } \\
\text { Group }\end{array}$ & value \\
\hline Age, yr, mean(SD) & $70.4(3.19)$ & $70.6(3.27)$ & 0.684 & $49.6(11.95)$ & $49.9(11.34)$ & 0.786 \\
\hline Men, $n(\%)$ & $97(66.0)$ & $93(64.6)$ & 0.802 & $126(70.0)$ & $127(70.6)$ & 0.908 \\
\hline $\mathrm{BMl}, \mathrm{kg} / \mathrm{m}^{2}$, mean $(\mathrm{sd})$ & $22.4(3.37)$ & $22.4(3.42)$ & 0.929 & $23.4(3.25)$ & $23.3(3.26)$ & 0.749 \\
\hline Systolic blood pressure, mm Hg, mean (sd) & $134.9(23.86)$ & $132.8(25.20)$ & 0.470 & $125.7(22.59)$ & $127.7(21.55)$ & 0.387 \\
\hline Heart rate, beats/min, mean (sd) & $105.3(19.28)$ & $106.7(20.86)$ & 0.564 & $111.7(19.59)$ & $113.5(20.05)$ & 0.387 \\
\hline Respiratory rate, breaths/min, mean (sd) & $28.0(6.02)$ & $27.2(6.32)$ & 0.270 & $28.1(6.43)$ & $28.9(6.26)$ & 0.256 \\
\hline Temperature, ${ }^{\circ} \mathrm{C}$, mean (sd) & $37.8(1.06)$ & $37.9(1.06)$ & 0.280 & $38.3(1.04)$ & $38.3(1.06)$ & 0.790 \\
\hline Pao2/Fio2, mean (sd) & $175.3(56.97)$ & $172.4(62.77)$ & 0.684 & $172.0(54.28)$ & $165.4(49.57)$ & 0.231 \\
\hline Glasgow score, mean (sd) & $11.3(3.70)$ & $11.3(3.35)$ & 0.922 & $11.7(3.42)$ & $11.8(3.51)$ & 0.915 \\
\hline \multicolumn{7}{|l|}{ Comorbidities, $n(\%)$} \\
\hline Diabetes mellitus (any type) & $7(4.76)$ & $5(3.47)$ & 0.580 & $1(0.56)$ & $6(3.33)$ & 0.121 \\
\hline Chronic bronchitis & $4(2.72)$ & $2(1.39)$ & 0.684 & $0(0.00)$ & $2(1.11)$ & 0.499 \\
\hline Coronary artery disease & $7(4.76)$ & $5(3.47)$ & 0.580 & $2(1.11)$ & $1(0.56)$ & $>0.999$ \\
\hline Hypertension & $31(21.09)$ & $32(22.22)$ & 0.814 & $14(7.78)$ & $8(4.44)$ & 0.056 \\
\hline Parkinson's disease & $2(1.36)$ & $0(0.00)$ & 0.498 & $1(0.56)$ & $1(0.56)$ & $>0.999$ \\
\hline Polytrauma & 0 & 0 & & $2(1.11)$ & $1(0.56)$ & $>0.999$ \\
\hline Etiological agents, $n(\%)^{\mathrm{a}}$ & $57(38.8)$ & $50(34.7)$ & 0.473 & $88(48.9)$ & $89(49.4)$ & 0.916 \\
\hline C-reactive protein, mg/L, mean (sd) & $87.6(95.49)$ & $80.9(69.68)$ & 0.531 & $87.5(84.52)$ & $90.5(112.06)$ & 0.801 \\
\hline Leucocytes, $10^{9}$ cells/L, mean (sd) & $12.1(5.98)$ & $12.4(6.16)$ & 0.747 & $13.0(7.04)$ & $14.1(7.34)$ & 0.159 \\
\hline Glucose, fasting morning, $\mathrm{mmol} / \mathrm{L}$ & $8.3(3.82)$ & $8.5(3.82)$ & 0.701 & $8.1(3.53)$ & $8.3(3.84)$ & 0.544 \\
\hline PSI score $\mathrm{a}, n(\%)$ & & & 0.741 & & & 0.462 \\
\hline Class II & $1(0.7)$ & $2(1.4)$ & & $19(10.6)$ & $20(11.1)$ & \\
\hline Class III & $18(12.2)$ & $13(9.0)$ & & 39 ( 21.7$)$ & $33(18.3)$ & \\
\hline Class IV & $80(54.4)$ & $78(54.2)$ & & $87(48.3)$ & $80(44.4)$ & \\
\hline Class V & $48(32.7)$ & $51(35.4)$ & & $35(19.4)$ & $47(26.1)$ & \\
\hline Total PSI score, mean $(\mathrm{sd})^{\mathrm{C}}$ & $122.9(28.99)$ & $125.1(31.41)$ & 0.521 & $106.4(29.17)$ & $108.9(32.79)$ & 0.445 \\
\hline Sequential Organ Failure Assessment (SOFA) score, mean (sd) & $5.9(2.66)$ & $5.9(2.59)$ & 0.838 & $6.3(2.85)$ & $6.3(2.98)$ & 0.986 \\
\hline $\begin{array}{l}\text { Acute Physiology and Chronic Health Evaluation II score, } \\
\qquad \text { mean (sd) }\end{array}$ & $17.2(6.35)$ & $16.6(5.88)$ & 0.389 & $14.9(5.93)$ & $15.0(6.14)$ & 0.944 \\
\hline
\end{tabular}




\begin{tabular}{|c|c|c|c|c|c|c|}
\hline Antibiotic susceptibility testing, $n(\%)^{\mathrm{d}}$ & $15(39.5)$ & $11(35.5)$ & 0.734 & $26(41.9)$ & $30(56.6)$ & 0.117 \\
\hline Mechanical ventilation, $n(\%)$ & $93(63.3)$ & $91(63.2)$ & 0.990 & 109 ( 60.6) & $111(61.7)$ & 0.829 \\
\hline
\end{tabular}

TABLE 2. Rate of Patients with Acute Respiratory Distress Syndrome and Septic Shock, the Baseline Settings of Mechanical Ventilation, and the Frequency of Antimicrobial Prescriptions for XueBiJing Group versus Placebo Group Using Descriptive Statistics for the Intention-to-Treat Populations 


\begin{tabular}{|c|c|c|c|c|c|c|}
\hline \multirow[t]{2}{*}{ Characteristics } & \multicolumn{3}{|c|}{ Elderly $\geq 65$} & \multicolumn{3}{|c|}{ Nonelderly $\square 65$} \\
\hline & Xuebijing Group & Placebo Group & $P$ value & Placebo Group & Xuebijing Group & $P$ value \\
\hline \multicolumn{7}{|l|}{ Septic shock and ARDS, $n(\%)$} \\
\hline Septic shock & $8(5.4)$ & $7(4.9)$ & 0.823 & $13(7.2)$ & $9(5.0)$ & 0.379 \\
\hline ARDS & $42(28.6)$ & $47(32.6)$ & 0.452 & $60(33.3)$ & $52(28.9)$ & 0.362 \\
\hline Mechanical ventilation, $n(\%)$ & $93(63.3)$ & $91(63.2)$ & & $109(60.6)$ & $111(61.7)$ & 0.829 \\
\hline Invasive ventilation, $n(\%)$ & $63(67.7)$ & $64(70.3)$ & 0.704 & $85(78.0)$ & $82(73.9)$ & 0.476 \\
\hline Tidal volume, mean (sd) & $457.0(58.62)$ & $463.9(74.80)$ & 0.486 & $469.9(76.24)$ & $474.8(92.12)$ & 0.667 \\
\hline Positive end expiratory pressure, mean (sd) & $6.0(2.16)$ & $6.3(2.90)$ & 0.398 & $6.6(2.76)$ & $6.4(2.72)$ & 0.750 \\
\hline $\mathrm{AC}, n(\%)$ & $6(6.5)$ & $9(9.9)$ & & $4(3.7)$ & $5(4.5)$ & \\
\hline Bi-level positive airway pressure ventilation, $n(\%)$ & $10(10.8)$ & $14(15.4)$ & & $17(15.6)$ & $17(15.3)$ & \\
\hline Continuous positive airway pressure, $n(\%)$ & $5(5.4)$ & $2(2.2)$ & & $3(2.8)$ & $4(3.6)$ & \\
\hline Noninvasive ventilation, $n(\%)$ & $21(22.6)$ & $11(12.1)$ & & $9(8.3)$ & $19(17.1)$ & \\
\hline $\mathrm{PC}, n(\%)$ & $8(8.6)$ & $8(8.8)$ & & $6(5.5)$ & $14(12.6)$ & \\
\hline PSV, $n(\%)$ & $6(6.5)$ & $9(9.9)$ & & $11(10.1)$ & $5(4.5)$ & \\
\hline SIMV, $n(\%)$ & $21(22.6)$ & $23(25.3)$ & & $50(45.9)$ & $38(34.2)$ & \\
\hline SIMV + PS, $n(\%)$ & $13(14.0)$ & $12(13.2)$ & & $6(5.5)$ & $7(6.3)$ & \\
\hline SIMV + SIMV/AS, $n(\%)$ & 0 & 0 & & $1(0.9)$ & $0(0.0)$ & \\
\hline SPMV + PS, $n(\%)$ & $3(3.2)$ & $3(3.3)$ & & $2(1.8)$ & $2(1.8)$ & \\
\hline \multicolumn{7}{|l|}{ Antimicrobial treatment, $n(\%)$} \\
\hline Beta-lactam & $143(97.28)$ & $141(97.92)$ & $>0.999$ & $172(95.56)$ & $170(94.44)$ & 0.629 \\
\hline Quinolones & $51(34.69)$ & $64(44.44)$ & 0.089 & $59(32.78)$ & $59(32.78)$ & 0.067 \\
\hline Glycopeptide & $30(20.41)$ & $20(13.89)$ & 0.141 & $29(16.11)$ & $50(27.78)$ & 0.008 \\
\hline Oxazolidinones & $18(12.24)$ & $17(11.81)$ & 0.908 & $31(17.22)$ & $19(10.56)$ & 0.067 \\
\hline Antifungal agents & $9(6.12)$ & $10(6.94)$ & 0.777 & $19(10.56)$ & $11(6.11)$ & 0.562 \\
\hline Tetracyclines & $8(5.44)$ & $7(4.86)$ & 0.823 & $15(8.33)$ & $12(6.67)$ & 0.548 \\
\hline Macrolide & $4(2.72)$ & $2(1.39)$ & $0.684^{\wedge}$ & $6(3.33)$ & $4(2.22)$ & 0.521 \\
\hline Aminoglycoside & $3(2.04)$ & $2(1.39)$ & $>0.999^{\wedge}$ & $1(0.56)$ & $2(1.11)$ & $>0.999$ \\
\hline Nitroimidazoles & $1(0.68)$ & $4(2.78)$ & $0.211^{\wedge}$ & $4(2.22)$ & $6(3.33)$ & 0.521 \\
\hline Antivirals & $2(1.36)$ & $1(0.69)$ & $>0.999^{\wedge}$ & $4(2.22)$ & $2(1.11)$ & 0.685 \\
\hline Lincomycin & $1(0.68)$ & $2(1.39)$ & $0.620^{\wedge}$ & $1(0.56)$ & $1(0.56)$ & $>0.999$ \\
\hline Sulfanilamide & $0(0.00)$ & $2(1.39)$ & $0.244^{\wedge}$ & $1(0.56)$ & $1(0.56)$ & $>0.999$ \\
\hline
\end{tabular}




\section{TABLE 3. The Primary and Two Secondary Outcomes}

\begin{tabular}{|c|c|c|c|c|c|c|}
\hline \multirow[t]{2}{*}{ Characteristics } & \multicolumn{3}{|c|}{ Elderly $\geq 65$} & \multicolumn{3}{|c|}{ Nonelderly 165} \\
\hline & Placebo Group & Xuebijing Group & $P$ value & Placebo Group & Xuebijing Group & $P$ value \\
\hline 28-d mortality, $n(\%)$ & $41(27.9)$ & $23(16.0)$ & 0.014 & $41(22.8)$ & $28(15.6)$ & 0.082 \\
\hline The time of mechanical ventilation, $d$, mean (std) & $14.3(8.97)$ & $13.1(8.80)$ & 0.422 & $10.8(8.27)$ & $10.3(8.46)$ & 0.679 \\
\hline Total duration of ICU stay, d, mean (std) & $15.6(7.88)$ & $13.9(7.84)$ & 0.133 & $12.3(7.41)$ & $11.8(7.67)$ & 0.581 \\
\hline
\end{tabular}

TABLE S1. Microbiologic identification for XueBiJing group vs placebo group using descriptive statistics for the intention-to-treat populations

\begin{tabular}{|c|c|c|c|c|c|c|c|}
\hline \multicolumn{2}{|c|}{ Characteristics } & \multicolumn{3}{c|}{ Elderly $\geq 65$} & \multicolumn{3}{c|}{ Nonelderly 065} \\
\hline \multicolumn{2}{|c|}{} & Placebo Group & Xuebijing Group & P value & Placebo Group & Xuebijing Group & P value \\
\hline Microbiologic identification & Yes(\%) & $57(38.8)$ & $50(34.7)$ & 0.473 & $89(49.4)$ & $88(48.9)$ & 0.916 \\
\hline Etiology & Known(\%) & $38(66.7)$ & $31(62.0)$ & 0.615 & $53(59.6)$ & $62(70.5)$ & 0.128 \\
\hline Sensitivity tests & Known(\%) & $15(39.5)$ & $11(35.5)$ & 0.734 & $30(56.6)$ & $26(41.9)$ & 0.117 \\
\hline
\end{tabular}


Table S2. Comparison of the secondary outcomes for the intention-to-treat populations

\begin{tabular}{|c|c|c|c|c|c|c|}
\hline \multirow[t]{2}{*}{ Characteristics } & \multicolumn{3}{|c|}{ Elderly $\geq 65$} & \multicolumn{3}{|c|}{ Nonelderly 065} \\
\hline & Placebo Group & Xuebijing Group & Pvalue & Placebo Group & Xuebijing Group & P value \\
\hline \multicolumn{7}{|c|}{ PSI score, mean(SD) } \\
\hline Day 4 & $108.6(29.16)$ & $102.5(26.13)$ & 0.069 & $90.8(26.56)$ & $83.2(28.14)$ & 0.022 \\
\hline Day 8 & $106.9(28.92)$ & $96.0(26.18)$ & 0.001 & $84.8(28.71)$ & $78.3(31.21)$ & 0.093 \\
\hline \multicolumn{7}{|c|}{ Sequential Organ Failure Assessment Score, mean(SD) } \\
\hline Day 4 & $5.3(2.88)$ & $4.5(2.66)$ & 0.017 & $4.9(2.92)$ & $4.6(3.20)$ & 0.350 \\
\hline Day 8 & $4.9(2.71)$ & $3.7(2.76)$ & $<0.001$ & $4.2(3.15)$ & $3.7(3.25)$ & 0.139 \\
\hline \multicolumn{7}{|c|}{ APACHE II score, mean (SD) } \\
\hline Day 4 & $5.6(3.58)$ & $3.9(3.06)$ & $<0.001$ & $5.5(3.80)$ & $4.8(4.23)$ & 0.112 \\
\hline Day 8 & $4.9(3.47)$ & $3.6(3.33)$ & 0.001 & $5.0(4.18)$ & $4.1(4.51)$ & 0.069 \\
\hline \multicolumn{7}{|c|}{ Lung Injury Score, mean(SD) } \\
\hline Day 4 & $7.5(2.37)$ & $6.5(2.06)$ & 0.006 & $7.3(2.88)$ & $7.2(2.72)$ & 0.809 \\
\hline Day 8 & $7.4(2.75)$ & $6.4(2.18)$ & 0.029 & $7.8(3.02)$ & $7.4(2.48)$ & 0.442 \\
\hline \multicolumn{7}{|c|}{ MODS score, mean (SD) } \\
\hline Day 4 & $6.3(3.20)$ & $5.9(3.00)$ & 0.596 & $5.0(2.69)$ & $5.1(3.64)$ & 0.769 \\
\hline Day 8 & $6.2(2.90)$ & $4.7(3.41)$ & 0.056 & $4.1(3.30)$ & $3.9(4.10)$ & 0.790 \\
\hline \multicolumn{7}{|c|}{ Body temperature, mean (SD) } \\
\hline Day 4 & $37.0(0.69)$ & $36.9(0.59)$ & 0.212 & $37.4(0.84)$ & $37.3(0.72)$ & 0.125 \\
\hline Day 8 & $36.9(0.68)$ & $36.8(0.50)$ & 0.139 & $37.2(0.78)$ & $37.0(0.62)$ & 0.048 \\
\hline \multicolumn{7}{|c|}{ Oxygenation index, mean (SD) } \\
\hline Day 4 & $222.8(87.66)$ & $251.1(96.38)$ & 0.011 & $232.2(98.72)$ & $245.3(101.90)$ & 0.238 \\
\hline Day 8 & $243.6(91.48)$ & $275.1(98.08)$ & 0.007 & $258.7(115.69)$ & $265.1(112.26)$ & 0.619 \\
\hline \multicolumn{7}{|l|}{ Procalcitonin } \\
\hline Day 4 & $4.5(20.22)$ & $5.6(19.24)$ & 0.680 & $5.2(16.26)$ & $5.7(20.29)$ & 0.837 \\
\hline Day 8 & $1.7(4.07)$ & $2.7(6.64)$ & 0.219 & $7.6(37.27)$ & $2.5(8.15)$ & 0.133 \\
\hline \multicolumn{7}{|l|}{ C-reactive protein } \\
\hline Day 4 & $65.2(61.37)$ & $57.3(56.35)$ & 0.313 & $64.1(64.31)$ & $62.4(76.32)$ & 0.857 \\
\hline Day 8 & $51.1(92.29)$ & $41.6(51.11)$ & 0.356 & $46.9(66.27)$ & $34.9(47.25)$ & 0.114 \\
\hline D-dimer & & & & & & \\
\hline
\end{tabular}




\begin{tabular}{|c|c|c|c|c|c|c|}
\hline Day 4 & $2137(2378.1)$ & $2423(2945.5)$ & 0.433 & $2534(4142.3)$ & $4365(7558.4)$ & 0.019 \\
\hline Day 8 & $2378(3106.4)$ & $2474(3599.1)$ & 0.836 & $2552(4837.7)$ & $3121(4762.3)$ & 0.358 \\
\hline Systemic Inflammatory Response Syndrome (SIRS) score, mean (sd) & & \multicolumn{4}{|l|}{} \\
\hline Day 4 & $2.0(1.08)$ & $1.6(1.11)$ & 0.002 & $2.2(1.16)$ & $2.0(1.27)$ & 0.152 \\
\hline Day 8 & $1.6(1.22)$ & $1.2(1.10)$ & 0.005 & $1.8(1.28)$ & $1.5(1.25)$ & 0.018 \\
\hline Total duration of antibiotic use, days, mean(SD) & $10.3(4.58)$ & $9.5(4.47)$ & 0.143 & $9.0(4.61)$ & $9.4(4.80)$ & 0.351 \\
\hline
\end{tabular}

TABLE S3. Adverse events and Clinically Significant Laboratory Abnormalities 


\begin{tabular}{|c|c|c|c|c|}
\hline \multirow[t]{2}{*}{ Characteristics } & \multicolumn{2}{|c|}{ Elderly $\geq 65$} & \multicolumn{2}{|c|}{ Nonelderly 065} \\
\hline & Placebo Group & Xuebijing Group & Placebo Group & Xuebijing Group \\
\hline Clinically Significant Laboratory Abnormalities & $64(43.54)$ & $55(38.19)$ & $68(37.78)$ & $61(33.89)$ \\
\hline Elevated alanine aminotransferase & $18(12.24)$ & $12(8.33)$ & $12(6.67)$ & $18(10.00)$ \\
\hline Elevated aspartate amino transferase & $13(8.84)$ & $11(7.64)$ & $5(2.78)$ & $10(5.56)$ \\
\hline Elevated blood urea nitrogen & $8(5.44)$ & $12(8.33)$ & $11(6.11)$ & $8(4.44)$ \\
\hline Elevated glucose & $8(5.44)$ & $7(4.86)$ & $7(3.89)$ & $11(6.11)$ \\
\hline Low red blood cell count & $6(4.08)$ & $8(5.56)$ & $10(5.56)$ & $13(7.22)$ \\
\hline High white blood cell count & $6(4.08)$ & $6(4.17)$ & $8(4.44)$ & $10(5.56)$ \\
\hline Elevated total bilirubin & $8(5.44)$ & $4(2.78)$ & $4(2.22)$ & $5(2.78)$ \\
\hline Elevated serum creatinine & $6(4.08)$ & $5(3.47)$ & $4(2.22)$ & $2(1.11)$ \\
\hline Elevated prothrombin time & $6(4.08)$ & $3(2.08)$ & $5(2.78)$ & $1(0.56)$ \\
\hline Elevated plasma fibrinogen & $6(4.08)$ & $3(2.08)$ & $7(3.89)$ & $4(2.22)$ \\
\hline Low white blood cell count & $4(2.72)$ & $4(2.78)$ & $2(1.11)$ & $1(0.56)$ \\
\hline Elevated activated partial thromboplastin time & $5(3.40)$ & $1(0.69)$ & $3(1.67)$ & $2(1.11)$ \\
\hline Low hemoglobin count & $4(2.72)$ & $1(0.69)$ & $7(3.89)$ & $1(0.56)$ \\
\hline High platelet count & $3(2.04)$ & $2(1.39)$ & $4(2.22)$ & $3(1.67)$ \\
\hline Decreased K+ & $1(0.68)$ & $3(2.08)$ & $10(5.56)$ & $4(2.22)$ \\
\hline Elevated $\mathrm{Na}+$ & $2(1.36)$ & $2(1.39)$ & $3(1.67)$ & $2(1.11)$ \\
\hline Decreased $\mathrm{Na}+$ & $2(1.36)$ & $2(1.39)$ & $2(1.11)$ & $0(0.00)$ \\
\hline Elevated fibrin D-dimer & $3(2.04)$ & $1(0.69)$ & $3(1.67)$ & $4(2.22)$ \\
\hline Decreased serum creatinine & $2(1.36)$ & $2(1.39)$ & $5(2.78)$ & $3(1.67)$ \\
\hline Low white blood cell count & $1(0.68)$ & $2(1.39)$ & $2(1.11)$ & $1(0.56)$ \\
\hline Elevated K+ & $1(0.68)$ & $2(1.39)$ & & \\
\hline High urine white blood cell count & $2(1.36)$ & $1(0.69)$ & $1(0.56)$ & $2(1.11)$ \\
\hline Positive protein in urine & $1(0.68)$ & $2(1.39)$ & $0(0.00)$ & $1(0.56)$ \\
\hline High urine red blood cell count & $2(1.36)$ & $1(0.69)$ & $1(0.56)$ & $2(1.11)$ \\
\hline Elevated urine glucose & $3(2.04)$ & $0(0.00)$ & $1(0.56)$ & $2(1.11)$ \\
\hline Decreased AST & $1(0.68)$ & $2(1.39)$ & $0(0.00)$ & $1(0.56)$ \\
\hline Decreased blood urea nitrogen & $2(1.36)$ & $1(0.69)$ & & \\
\hline Abnormal alanine aminotransferase & $2(1.36)$ & $0(0.00)$ & & \\
\hline High red blood cell count & $2(1.36)$ & $0(0.00)$ & $1(0.56)$ & $1(0.56)$ \\
\hline
\end{tabular}




\begin{tabular}{|c|c|c|c|c|}
\hline Decreased plasma fibrinogen & $1(0.68)$ & $1(0.69)$ & $2(1.11)$ & $1(0.56)$ \\
\hline Decreased total bilirubin & $0(0.00)$ & $2(1.39)$ & $1(0.56)$ & $0(0.00)$ \\
\hline Positive fecal occult blood test & & & $1(0.56)$ & $0(0.00)$ \\
\hline Elevated $\mathrm{C}$ reactive protein & $1(0.68)$ & $0(0.00)$ & & \\
\hline Decreased activated partial thromboplastin time & $1(0.68)$ & $0(0.00)$ & & \\
\hline Elevated procalcitonin & $1(0.68)$ & $0(0.00)$ & & \\
\hline Decreased prothrombin time & $0(0.00)$ & $1(0.69)$ & & \\
\hline High hemoglobin count & $1(0.68)$ & $0(0.00)$ & & \\
\hline
\end{tabular}

\section{References}

1. Brown RB: Community-acquired pneumonia: diagnosis and therapy of older adults. Geriatrics 1993, 48(2):43-50.

2. Loeb MB: Community-acquired pneumonia in older people: the need for a broader perspective. Journal of the American Geriatrics Society 2003, 51(4):539-543.

3. Neupane B, Walter SD, Krueger P, Marrie T, Loeb M: Predictors of inhospital mortality and re-hospitalization in older adults with community-acquired pneumonia: a prospective cohort study. BMC geriatrics 2010, 10:22.

4. Kelly E, MacRedmond RE, Cullen G, Greene CM, McElvaney NG, O'Neill SJ: Community-acquired pneumonia in older patients: does age influence systemic cytokine levels in community-acquired pneumonia? Respirology 2009, 14(2):210-216.

5. Vila-Corcoles A, Ochoa-Gondar O, Rodriguez-Blanco T, Raga-Luria X, Gomez-Bertomeu F: Epidemiology of community-acquired pneumonia in older adults: a population-based study. Respiratory medicine 2009, 103(2):309-316.

6. Fung HB, Monteagudo-Chu MO: Community-acquired pneumonia in the elderly. The American journal of geriatric pharmacotherapy 2010, 8(1):47-62.

7. Mandell LA, Wunderink RG, Anzueto A, Bartlett JG, Campbell GD, Dean NC, Dowell SF, File TM, Jr., Musher DM, Niederman MS et al: Infectious Diseases Society of America/American Thoracic Society consensus guidelines on the management of community-acquired pneumonia in adults. Clinical infectious diseases : an official publication of the Infectious Diseases Society of America 2007, 44 Suppl 2:S27-72.

8. Fleischmann C, Scherag A, Adhikari NK, Hartog CS, Tsaganos T, Schlattmann P, Angus DC, Reinhart K: Assessment of Global Incidence and Mortality of Hospital-treated Sepsis. Current Estimates and Limitations. Am J Respir Crit Care Med 2016, 193(3):259-272.

9. Qi F, Liang ZX, She DY, Yan GT, Chen LA: A clinical study on the effects and mechanism of xuebijing injection in severe pneumonia patients. Journal of traditional Chinese medicine = Chung i tsa chih ying wen pan 2011, 31(1):46-49.

10. Li C, Wang P, Zhang L, Li M, Lei X, Liu S, Feng Z, Yao Y, Chang B, Liu B: Efficacy and safety of Xuebijing injection (a Chinese patent) for sepsis: a meta-analysis of randomized controlled trials. J Ethnopharmaco/2018.

11. Jiang M, Zhou M, Han Y, Xing L, Zhao H, Dong L, Bai G, Luo G: Identification of NF-kB Inhibitors in Xuebijing injection for sepsis treatment based on bioactivity-integrated UPLC-Q/TOF. J Ethnopharmaco/ 2013, 147(2):426- 
433.

12. Wang Q, Wu X, Tong X, Zhang Z, Xu B, Zhou W: Xuebijing Ameliorates Sepsis-Induced Lung Injury by Downregulating HMGB1 and RAGE Expressions in Mice. Evidence-Based Complementray and Alternative Medicine,2015,(2015-3-2) 2015, 2015(264):860259.

13. Liu Y, Tian X, Cui M, Zhao S: Safflower yellow inhibits angiotensin Il-induced adventitial fibroblast proliferation and migration. Journal of Pharmacological Sciences 2014, 126(2):107.

14. Song Y, Yao C, Yao Y, Han H, Zhao X, Yu K, Liu L, Xu Y, Liu Z, Zhou Q et al: XueBiJing Injection Versus Placebo for Critically III Patients With Severe Community-Acquired Pneumonia: A Randomized Controlled Trial. Crit Care Med 2019, 47(9):e735-e743.

15. Fine MJ, Smith DN, Singer DE: Hospitalization decision in patients with community-acquired pneumonia: a prospective cohort study. The American journal of medicine 1990, 89(6):713-721.

16. Marrie TJ, Haldane EV, Faulkner RS, Durant H, Kwan C: Community-acquired pneumonia requiring hospitalization. Is it different in the elderly? Journal of the American Geriatrics Society 1985, 33(10):671-680.

17. Kaplan V, Angus DC, Griffin MF, Clermont G, Scott Watson R, Linde-Zwirble WT: Hospitalized community-acquired pneumonia in the elderly: age- and sex-related patterns of care and outcome in the United States. Am J Respir Crit Care Med 2002, 165(6):766-772.

18. Rello J, Rodriguez R, Jubert P, Alvarez B: Severe community-acquired pneumonia in the elderly: epidemiology and prognosis. Study Group for Severe Community-Acquired Pneumonia. Clinical infectious diseases : an official publication of the Infectious Diseases Society of America 1996, 23(4):723-728.

19. Venkatesh B, Finfer S, Cohen J, Rajbhandari D, Arabi Y, Bellomo R, Billot L, Correa M, Glass P, Harward M et al: Adjunctive Glucocorticoid Therapy in Patients with Septic Shock. The New England journal of medicine 2018, 378(9):797-808.

20. Agbabiaka T, Wider B, Watson LK, Goodman C: Concurrent use of prescription drugs and herbal medicinal products in older adults: a systematic review protocol. Syst Rev 2016, 5:65.

\section{Figures}

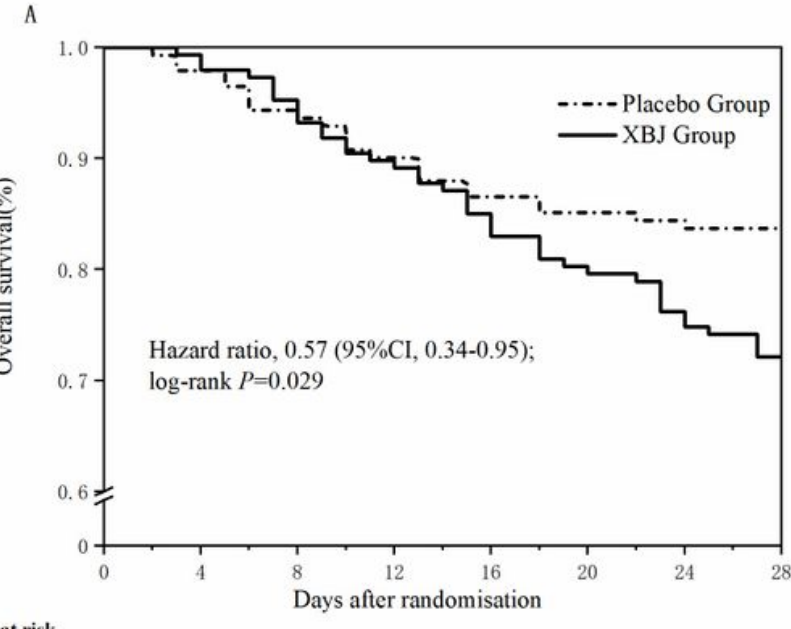

Number at risk

$\begin{array}{lllllllll}\text { XBJ group } & 144 & 138 & 133 & 127 & 122 & 120 & 119 & 118\end{array}$

$\begin{array}{lllllllll}\text { Placebo group } & 147 & 146 & 140 & 132 & 125 & 118 & 112 & 106\end{array}$

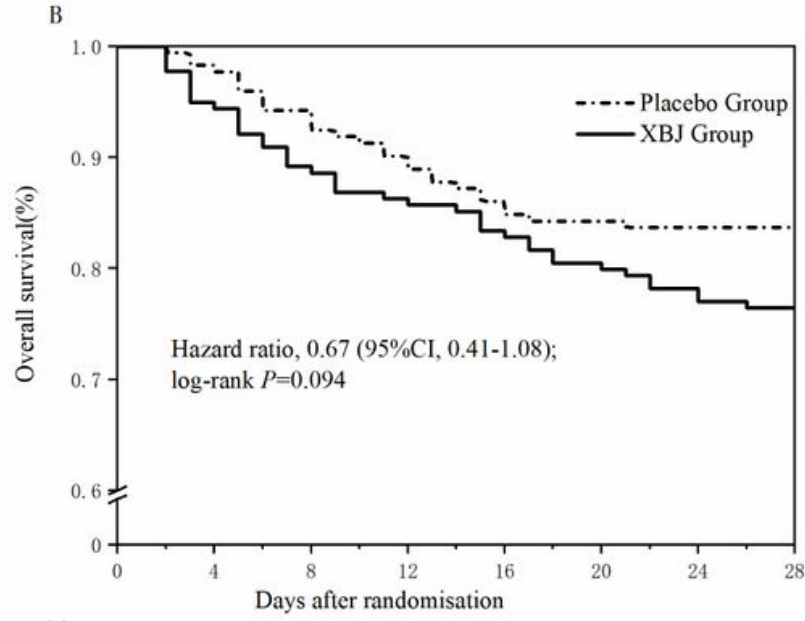

Number at risk

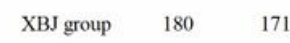
Placebo group 180 


\section{Figure 1}

Kaplan-Meier (K-M) survival curve of overall survival after XueBiJing (XBJ) and placebo for patients in (A) elderly group: Patients with XBJ had a significantly inferior overall survival to those who with placebo. The HR of was 0.57 (95\% Cl, 0.34-0.95; $\mathrm{P}=0$.029), (B) nonelderly group: The HR of was 0.67 (95\% Cl, 0.41-1.08; $P=0.0949$ ). 